\title{
Nematóide de galha em rabanete: suscetibilidade de cultivares e patogenicidade.
}

\author{
Carlos Eduardo Rossi; Pollyanna T. Montaldi
}

Instituto Biológico, Centro de Pesquisa e Desenvolvimento em Sanidade Vegetal, C. Postal 70, 13001-970 Campinas-SP. E-mail: crossi@biologico.br

\section{RESUMO}

Pesquisaram-se as reações de 11 cultivares de rabanete a Meloidogyne javanica e sua patogenicidade a uma dessas cultivares. No estudo de reações, os tratamentos/cultivares foram 'Comprido Branco', 'Crimson Gigante', 'Ponta Branca', 'Comprido Vermelho', 'Serrano', 'Gigante Wurzburgo', 'Saxa', 'Redondo Verme1ho', 'Redondo Gigante', 'Cometo' e 'Akamaru Hatsuka'. Determinaram-se os índices de galhas (IG) e de massas de ovos (IMO), o número de nematóides no sistema radicular (NSR) e por grama de raiz (NGR) e o fator de reprodução (FR) 53 dias após a inoculação artificial com 2.000 ovos do parasito. Em outro experimento, avaliaram-se os efeitos de três densidades populacionais do nematóide [0, 500 (nível populacional baixo) e 10.000 (nível populacional alto) ovos/planta] sobre as massas frescas e secas de túberas e de parte aérea da cultivar 'Redondo Gigante', 39 dias após a inoculação. Os resultados mostraram que todas as cultivares permitiram a reprodução de $M$. javanica, sendo portanto consideradas suscetíveis. Valores de IG e IMO foram maiores ou iguais a 2,5 e os de FR, maiores do que 8,0 para todas as cultivares estudadas. O parasito causou diminuição significativa nas massas frescas e secas de túberas e de partes aéreas nos dois níveis populacionais estudados comparados com o controle não inoculado. As médias dos tratamentos contendo níveis populacionais baixo e alto do nematóide também diferiram estatisticamente entre si, comprovando-se, assim, a sua ação patogênica sobre a cultivar avaliada.

Palavras-chave: Meloidogyne javanica, Raphanus sativus, patogenicidade, reação de cultivares.

\begin{abstract}
Root-knot nematode: cultivars reaction and damage to radish

Experiments were conducted under greenhouse conditions to determine the reaction of eleven radish (Raphanus sativus) cultivars to Meloidogyne javanica and the pathogenicity of this nematode to a previously selected cultivar. The cultivars tested were 'Comprido Branco', 'Crimson Gigante', 'Ponta Branca', 'Comprido Vermelho', 'Serrano', 'Gigante Wurzburgo', 'Saxa', 'Redondo Vermelho', 'Redondo Gigante', 'Cometo' and 'Akamaru Hatsuka'. Radish plants were inoculated with 2,000 eggs, and the [root-gall (IG) and eggmass (IMO) indexes and reproduction factor (FR)] were determined 53 days after inoculation. In the other experiment, three populational levels (0,500 (low level) and 10,000 (high level) nematode eggs) were individually inoculated in 'Redondo Vermelho' radish cultivar. The results showed values of IG and IMO superior or equal to 2.5 and FR superior to 8.0 and, according tp these data, all tested cultivars were classified as susceptible. Meloidogyne javanica caused plant growth supression as measured by the values of fresh and dry weight of roots and the foliage of the plants, 39 days after inoculations. The average treatments containing low and high populational levels were satisfically different among themselves, thus proving its patogenic action on the cultivars evaluated.
\end{abstract}

Keywords: Meloidogyne javanica, Raphanus sativus, pathogenicity, cultivar reaction.

\section{(Recebido para publicação em 25 de novembro de 2002 e aceito em 3 de novembro de 2003)}

$\mathrm{O}$ $s$ nematóides de galha (Meloidogyne spp.) são parasitos importantes para as hortaliças, causando-lhes sérios prejuízos. Meloidogyne javanica é uma espécie-chave por ser polífaga e muito bem adaptada aos agroecossistemas tropicais (Netscher e Sikora, 1990).

Hortaliças, como o rabanete (Raphanus sativus L.), de ciclo produtivo inferior ao do parasito, onde é comum a colheita de túberas após 20 a 30 dias da emergência (Camargo, 1984), podem ser manejadas de diferentes formas visando diminuir níveis populacionais do nematóide no solo (Brody e Laughlin, 1977; Gardner e Caswell-Chen, 1994; Johnson, 1998; Cuadra et al., 2000).
Segundo dados de literatura, o rabanete é considerado hospedeiro suscetível ao nematóide. Sasser (1954) verificou que $R$. sativus 'Early Scarlet Globe' foi passível de altas infecções, podendo apresentar abundante número de fêmeas e massas de ovos de $M$. arenaria, $M$. incognita e $M$. javanica. Trabalhos de Ahuja e Muchopadhyaya (1985), Paruthi et al. (1986), Kanwar et al. (1995), McSorley e Frederick (1995) e Carneiro et al. (2000) também corroboram essas observações. Entretanto, resultados divergentes foram encontrados por outros autores, demonstrando a resistência ou reduzida eficiência como hospedeira de algumas cultivares
(Calinga e Ballon, 1974; Castillo e Bulag, 1974; Reyes e Villanueva, 1981).

No Brasil, há citações nesta espécie vegetal da ocorrência de Meloidogyne incognita no Rio Grande do Norte (Ponte et al., 1977) e em São Paulo (Silveira et al., 1986).

Em vista da escassez de trabalhos publicados na área de nematologia em rabanete e procurando, preliminarmente, adequar a planta na estratégia de cultura armadilha de nematóides, estudaram-se as reações das principais cultivares disponíveis no mercado a Meloidogyne javanica e a sua patogenicidade a uma dessas cultivares. 
Tabela 1. Índice de galhas (IG) e de massa de ovos (IMO), nematóides no sistema radicular (NSR) e por grama de raiz (NGR) e fator de reprodução (FR) de Meloidogyne javanica e respectiva reação a cultivares de rabanete. Campinas, Instituto Biológico, 2001.

\begin{tabular}{|c|c|c|c|c|c|c|}
\hline Cultivar & IG' & $\mathrm{IMO}^{1}$ & NSR $^{2}$ & $\mathrm{NGR}^{2}$ & $\mathrm{FR}^{3}$ & Reação ${ }^{4}$ \\
\hline Saxa & 3,7 & 4,7 & 36250 & $13081,7 \mathrm{a}$ & 18,1 & $S$ \\
\hline Akamaru Hatsuka & 4,0 & 5,0 & 27160 & $10727,9 a$ & 13,6 & $S$ \\
\hline Crimson Gigante & 3,4 & 4,4 & 29520 & $8831,4 a b$ & 12,3 & $S$ \\
\hline Serrano & 3,0 & 3,8 & 27150 & $6198,9 \quad b$ & 10,9 & S \\
\hline Gigante Wurzburgo & 3,3 & 4,3 & 30025 & $6940,1 \quad b$ & 15,0 & $S$ \\
\hline Redondo Gigante & 3,2 & 4,0 & 16333 & $6659,0 \quad b$ & 8,2 & S \\
\hline Cometo & 2,5 & 3,5 & 20333 & $6353,6 \quad b$ & 10,2 & S \\
\hline Comprido Vermelho & 2,8 & 3,5 & 30520 & $5605,1 \quad b$ & 15,3 & $S$ \\
\hline Redondo Vermelho & 3,2 & 3,8 & 22600 & $5324,7 \quad b$ & 11,3 & $S$ \\
\hline Comprido Branco & 2,5 & 3,3 & 22150 & 4898,6 & 11,1 & $S$ \\
\hline Ponta Branca & 3,6 & 4,0 & 27175 & 5234,4 & 13,6 & $S$ \\
\hline Tomate 'Rutgers' & 5,0 & 5,0 & 82720 & 7185,3 & 41,4 & $S$ \\
\hline
\end{tabular}

${ }^{1}$ Baseado em proposta de Taylor \& Sasser (1978);

${ }^{2}$ Médias seguidas por letras distintas diferem entre si pelo teste de Stderr \& Pdiff a 5\%;

${ }^{3}$ Baseado em proposta de Oostenbrink (1966);

${ }^{4} \mathrm{~S}=$ suscetibilidade.

\section{MATERIAL E MÉTODOS}

\section{Reação de Cultivares}

Instalou-se o experimento em casa de vegetação provida de controle de temperatura, em delineamento inteiramente casualizado, com 6 repetições. Cada parcela constituiu-se num copo plástico com capacidade para $535 \mathrm{ml}$ preenchido com $500 \mathrm{ml}$ de substrato (solo e areia, 1:1) previamente desinfestado com brometo de metila $(150 \mathrm{ml} / \mathrm{m})$ e contendo uma planta. Os tratamentos (cultivares com respectivas empresas fornecedoras entre parêntesis) testados foram: 'Comprido Branco' (Horticeres), 'Crimson Gigante' (Ferti Seed), 'Ponta Branca' (Cal Seed), 'Comprido Vermelho' (Cal Seed), 'Serrano' (Feltrin), 'Gigante Wurzburgo' (Isla), 'Saxa' (Feltrin), 'Redondo Vermelho' (Horticeres), 'Redondo Gigante' (Feltrin), 'Cometo' (Topseed) e 'Akamaru Hatsuka' (Sakata). Utilizaram-se tomateiros 'Rutgers' para confirmação da viabilidade do inóculo. Nove dias após a semeadura e seis do desbaste, inocularam-se, individualmente, 2.000 ovos do parasito (Pi) extraídos de raízes de tomateiros 'Rutgers' cultivados em substrato artificialmente infestado, seguindo metodologia propos- ta por Hussey e Barker (1973) modificada por Boneti e Ferraz (1981). As plantas foram irrigadas diariamente e adubadas quinzenalmente com $20 \mathrm{ml} /$ planta de fertilizante líquido (6-6-8) diluído 1:250. Controlou-se a temperatura da casa de vegetação para não exceder $30^{\circ} \mathrm{C}$

Cinquenta e três dias após as inoculações, desenvasaram-se as plantas e, com auxílio de uma tesoura, separaram-se os prolongamentos da raiz principal das túberas; esses prolongamentos de raízes foram lavados sob água corrente e deixados para secar por 30 minutos à sombra, determinando-se os valores de massa fresca. Para evidenciar as massas de ovos externas foram feitas colorações com 'phloxine B', determinando-se também os números de galhas e de massas de ovos, obtendo-se os índices de galhas (IG) e os de massas de ovos (IMO), conforme escala de 0 a 5 , sendo $0=0$ galhas ou massas de ovos; $1=1-2 ; 2=3-10 ; 3=11-30 ; 4=31-100$ e $5=>100$ (Taylor e Sasser, 1978).

Para determinar o número de nematóides (ovos + juvenis de segundo estádio) no sistema radicular (NSR ou Pf) e por grama de raiz (NGR), processaram-se os sistemas radiculares pela técnica de Boneti e Ferraz (1981), estimando-se tais parâmetros em alíquotas de $1 \mathrm{ml}$ colocadas em lâmina de Peters e examinadas ao microscópio óptico. Analisaram-se estatisticamente os dados de NSR e NGR, aplicando-se o teste F na análise da variância e o teste de Stderr e Pdiff a 5\% para comparação de médias. Calculou-se o fator de reprodução (FR) pela razão: Pi/Pf (Oostenbrink, 1966), e determinando-se a reação das cultivares.

\section{Patogenicidade}

Como descrito para o anterior, esse experimento também foi instalado em casa de vegetação, em delineamento inteiramente casualizado, com seis repetições. Somente a parcela foi constituída de um recipiente plástico com capacidade para $2 \mathrm{~L}$ contendo 1,8 L do mesmo substrato e uma planta da cultivar 'Redondo Vermelho', selecionada com base em resultados do experimento anterior. Plântulas com um dia de idade foram inoculadas, separadamente no momento do transplantio, com três densidades populacionais do nematóide 0 , 500 e 10.000 ovos/planta. Esses valores numéricos foram qualificados como controle não inoculado e níveis populacionais baixo e alto, respectivamente. Tomateiros 'Rutgers' foram inoculados com 5.000 ovos para verificação da viabilidade do inóculo. Trinta e nove dias após a inoculação, 
Tabela 2. Efeito de Meloidogyne javanica nas massas frescas e secas de parte aérea (MFPA e MSPA) e túbera (MFT e MSPA) de rabanete 'Redondo Gigante'. Campinas, Instituto Biológico, 2001.

\begin{tabular}{lccccc}
\hline \multirow{2}{*}{$\begin{array}{c}\text { Nível } \\
\text { Populacional }\end{array}$} & \multicolumn{2}{c}{ Massa fresca $(\mathbf{g})$} & \multicolumn{2}{c}{ Massa seca $(\mathbf{g})$} & \multirow{2}{*}{ IG } \\
\cline { 2 - 5 } & Parte aérea & Túbera & Parte aérea & Túbera & \\
\hline Controle & $18,92 \mathrm{a}$ & $26,5 \mathrm{a}$ & $2,1 \mathrm{a}$ & $2,5 \mathrm{a}$ & 0 \\
Baixo $(5001)$ & $18,8 \mathrm{~b}$ & $17,6 \mathrm{~b}$ & $2,0 \mathrm{~b}$ & $1,6 \mathrm{~b}$ & 3,5 \\
Alto $(10000)$ & $15,9 \mathrm{c}$ & $6,7 \mathrm{c}$ & $1,7 \mathrm{c}$ & $0,6 \mathrm{c}$ & 4,8 \\
\hline
\end{tabular}

${ }^{1}$ Nível de inóculo inicial;

${ }^{2}$ Médias seguidas por letras distintas em cada coluna diferem entre si pelo teste de Tukey, ao nível de 5\% de probabilidade (Médias de 6 repetições. Valores transformados em raiz quadrada de $\mathrm{x}$ ).

desenvasaram-se as plantas e separaram-se os sistemas radiculares (prolongamentos da raiz principal e túberas) das partes aéreas com auxílio de uma tesoura; as raízes foram lavadas em água corrente e deixadas para secar por $30 \mathrm{mi}$ nutos à sombra, determinando-se os valores de massa fresca dos sistemas radiculares e das partes aéreas. Tanto os sistemas radiculares como os órgãos aéreos de cada planta foram colocados em sacos especiais de papel e postos para secar em estufa a $68-70^{\circ} \mathrm{C}$, anotando-se os respectivos valores de massa seca.

Para estimar a quantidade de nematóides remanescentes, uma muda de tomate 'Rutgers' foi transplantada para cada parcela, de onde tinham sido retiradas as plantas de rabanete. Vinte e quatro dias após o transplante, avaliaram-se os índices de galhas, segundo Taylor e Sasser (1978).

Os valores referentes às massas frescas e secas foram analisados estatisticamente, aplicando-se o teste F na análise da variância e o teste de Tukey a $5 \%$, no caso de ter ocorrido diferenças significativas entre médias.

\section{RESULTADOS E DISCUSSÃO}

\section{Reação de Cultivares}

Meloidogyne javanica se reproduziu em todas as cultivares estudadas, verificando-se para todas elas IG e IMO maiores ou iguais a 2,5, o que as caracteriza como suscetíveis segundo proposta de Canto-Saénz (1983) modificada por Sasser et al. (1984) (Tabela 1). Os valores de IG e IMO variaram de 2,53,3 para 'Cometo' e 2,5-3,5 para 'Comprido Branco' a 4,0-5,0 para 'Akamaru Hatsuka'. As galhas, não muito conspí- cuas, projetavam-se pelo prolongamento da raiz principal e nas raízes secundárias. Parece que essa característica é comum em Brassicaceae, pois Ferraz e Rossi (1999) já o haviam observado em raízes de canola. Não se verificou reação hiperplástica nas túberas. Fato semelhante foi observado por Brody e Laughlin (1977) para M. hapla. Não se verificaram diferenças estatísticas entre as cultivares para NSR. Porém, para NGR, a cultivar Saxa diferiu estatisticamente de todas as outras, com exceção de 'Akamaru Hatsuka' e 'Crimson Gigante', apresentando a maior média $(13.081,7)$. Entretanto, todas as cultivares mostraram-se com FR maior do que oito, o que evidencia mais uma vez a sua alta suscetibilidade ao nematóide.

Resultados semelhantes foram obtidos por Gardner e Caswell-Chen (1994), que demonstraram a suscetibilidade de sete cultivares de Raphanus sativus var. oleifera e por McSorley e Frederick (1995) que avaliaram a reação da cultivar Cherry Belle a $M$. javanica, entre outros nematóides, e também puderam demonstrar sua suscetibilidade. Carneiro et al. (2000), trabalhando com as cultivares Crison Gigante (provavelmente a mesma cultivar aqui estudada com o nome de 'Crimson Gigante') e Scarlet Globe, obtiveram valores de FR pouco maiores do que um, ou seja, relativamente menores do que os desse experimento, classificando-as como moderadamente suscetíveis. As diferenças observadas podem ser atribuídas às diferenças na metodologia de avaliação, já que os autores trabalharam com 5.000 ovos contra 2.000 desse experimento e período de avaliação de 3 meses contra 39 dias do presente trabalho.
Discordantemente, estudos realizados nas Filipinas com outra espécie de nematóide de galha ( $M$. incognita), demonstraram que as cultivares Early Scarlet Globe, Ramgo e outra não mencionada foram resistentes a esse parasito (Calinga e Ballon, 1974; Castillo e Bulag, 1974; Reyes e Villanueva, 1981). Fato ainda mais curioso foi descrito por Paruthi et al. (1986) que não observaram nenhuma massa de ovos nas raízes da cultivar H.R.I. após 90 dias da inoculação com seis níveis populacionais de $M$. javanica (0 a 10.000 juvenis/vaso).

\section{Patogenicidade}

O parasito causou diminuição significativa nas massas frescas e secas de túbera e de parte aérea do rabanete ' $\mathrm{Re}$ dondo Vermelho', nos dois níveis populacionais estudados (500 e 10.000) comparados com o tratamento que não recebeu inóculo (Tabela 2). As médias dos tratamentos dos níveis populacionais baixo e alto também diferiram significativamente entre si para todas as variáveis. Isso demonstra que, mesmo em pequena quantidade, o nematóide ocasiona dano ao rabanete, embora esse dano se intensifique com o aumento da sua população. Uma evidência adicional a esse fato são os altos IG observados: 3,5 para o nível baixo e 4,8, para o alto (Tabela 2). Para o caso dos níveis populacionais altos, cinco das seis repetições apresentavam-se com os sistemas radiculares contendo aglomerados de galhas e fortemente atrofiados, sendo portanto caracterizadas como nota 5. Paruthi et al. (1986) verificaram reduções nos valores de algumas variáveis testadas sob diferentes níveis de inóculo de M. javanica. A partir de 100 juvenis houve reduções significativas nas mas- 
sas frescas de parte aérea, mas somente acima de 2.000 juvenis/planta ocorreram reduções na massa de túberas.

Pesquisando a reação dessa hortaliça a M. incognita, Ahuja e Muchopadhyaya (1985) verificaram crescimento irregular e intensa redução no porte de plantas, com Pi variando de 5.000 a 10.000 juvenis de segundo estádio por planta.

Ainda no presente experimento, resultados de bio-ensaio com o tomateiro 'Rutgers', demonstraram que altas populações do nematóide também permanecem no substrato após a retirada das plantas, pelos valores de IG obtidos (4,7 e 4,3 para níveis baixo e alto, respectivamente). Assim, a sugestão de Brody e Laughlin (1977) que o rabanete poderia servir como planta armadilha para M. hapla deve ser melhor estudada para M. javanica. Mesmo assim, Cuadra et al. (2000) verificaram diminuições de $50 \%$ na população de $M$. incognita raça 2 no solo e incremento de 10 a $20 \%$ na produção de tomate, pepino e feijão caupi após uso da estratégia de cultura armadilha. Recomenda-se como práticas complementares a retirada e destruição de plantas cujas túberas não se desenvolveram adequadamente, portanto não tendo sido comercializadas, para evitar aumento da população do nematóide.

\section{LITERATURA CITADA}

AHUJA, S.; MUCHOPADHYAYA, M.C. Effect of nematode populations of Meloidogyne incognita on their reproduction and growth of radish and carrot. Bulletin of Entomology, v.26, n.2, p.214-217, 1985.

BONETI, J.I.S.; FERRAZ, S. Modificação do método de Hussey \& Barker para extração de ovos de Meloidogyne exigua de raízes de cafeeiro. Fitopatologia Brasileira, Brasília, v.6, p.553, 1981

BRODY, J.K.; LAUGHLIN, C.W. The effect of vegetable cropping sequences on population development of Meloidogyne hapla. Revista de Agricultura, v.52, n.1, p.13-18, 1977

CALINGA, R.H.; BALLON, F.B. Studies on the pathologic reactions of different varieties of vegetables to Meloidogyne incognita. Philippine Journal of Plant Industry, v.39, n.2, p.107-114, 1974. CAMARGO, L.S. As hortaliças e seu cultivo. 2. Ed. Campinas: Fundação Cargill, 1984. 448 p.

CANTO-SAENZ, M. The nature of resistance to Meloidogyne incognita (Kofoid \& White, 1919) Chitwood, 1949. In: RESEARCH \& PLANNING CONFERENCE ON ROOT-KNOT NEMATODES, Meloidogyne spp., 3., Lima, 1982. Proceedings. Raleigh: North Carolina State University, 1983. p.160-165.

CARNEIRO, R.M.D.G.; RANDING, O.; ALMEIDA, M.R.A.; CAMPOS, A.D. Resistance of vegetable crops to Meloidogyne spp.: suggestion for a crop rotation system. Nematologia Brasilei$r a$, v.24, n.1, p.49-54, 2000.

CASTILLO, M.B.; BULAG, V.B. Identification, pathogenicity and host range of a root-knot nematode species attacking celery in La Trinidad, Benguet. Philippine Agriculturist, v.57, n.9-10, p.345-352, 1974.

CUADRA, R.; CRUZ, X.; FAJARDO, J.L. The use of short cycle crops as trap crops for the control of root-knot nematodes. Nematropica, v.30, n.2, p.241-246, 2000.

FERRAZ, L.C.C.B.; ROSSI, C.E. Reprodução de fitonematóides em cultivares de canola. Revista de Agricultura, v.74, n.2, p.179-190, 1999.

GARDNER, J.; CASWELL-CHEN, E.P. Raphanus sativus, Sinapis alba, and Fagopyrum esculentum as hosts to Meloidogyne incognita, Meloidogyne javanica, and Plasmodiophora brassicae. Journal of Nematology, v.26, n.4S, p.756-760, 1994.

HUSSEY, R.S.; BARKER, K.R. A comparison of methods of collecting inocula of Meloidogyne spp. including a new technique. Plant Disease Reporter, v.57, p.1025-1028, 1973.

JOHNSON, A.W. Vegetable crops. In: BARKER, KR; PEDERSON, G.A.; WINDHAM, G.L. Plant and nematode interactions. Madison: American Society of Agronomy, 1998. p.595-635.

KANWAR, R.S.; WALIA, K.K.; GUPTA, D.C.; JAIN, R.K. Host response of some cultivars of vegetable and spice crops against Meloidogyne species and effect of different inoculum levels of Meloidogyne javanica on spices. Haryana Agricultural University Journal of Research, v.25, n.1-2, p.57-60, 1995.

McSORLEY, R.; FREDERICK, J.J. Responses of some common cruciferae to root-knot nematodes. Journal of Nematology, v.27, n.4S, p.550-554, 1995.

NETSCHER, C.; SIKORA, R.A. Nematode parasites of vegetables. In: LUC, M.; SIKORA, R.A.; BRIDGE, J. Plant parasitic nematodes in subtropical and tropical agriculture. Wallingford: CAB International, 1990. p.181-235.

OOSTENBRINK, M. Major characteristics of the relation between nematodes and plants Mededelingen Landbouwhogeschool, v.66, p.1-46, 1966.

PARUTHI, I.J.; JAIN, R.K.; GUPTA, D.C. Investigations on pathogenicity of root-knot nematode Meloidogyne javanica on radish and turnip. International Nematology Network Newsletter, v.3, n.4, p.8-10, 1986.

PONTE, J.J.; FERNANDES, E.R.; SILVA, A.T. Plantas hospedeiras de Meloidogyne no Estado do Rio Grande do Norte (Brasil). In: REUNIÃO DE NEMATOLOGIA, 2., Piracicaba, 1977. Trabalhos apresentados. Publicação Sociedade Brasileira de Nematologia, n.2, p. 67-70, 1977.

REYES, T.T.; VILLANUEVA, L.M. Screening of crop varieties for resistance to Meloidogyne incognita: I. Vegetable varieties. In: RESEARCH \& PLANNING CONFERENCE ON ROOTKNOT NEMATODES, Meloidogyne spp., 3., Jakarta, 1981. Proceedings. Raleigh: North Carolina State University, 1981. p.76-81

SASSER, J.N. Identification and host-parasite relationships of certain root-knot nematodes (Meloidogyne spp.). College Park: University of Maryland/Agricultural Experiment Station, 1954. 31 p. (Bulletin Technical, A-77)

SASSER, J.N.; CARTER, C.C.; HARTMAN, K.M. Standartization of host suitability studies and reporting of resistance to root-knot nematodes. Raleigh: North Carolina State University Press/ USDA, 1984. 7 p.

SILVEIRA, S.G.P.; CURI, S.M.; STEFANINI, P.C. Nematóides de plantas detectados pela Seção de Nematologia do Instituto Biológico de São Paulo, Brasil. Biológico, v.52, n.10/12, p.91-104, 1986.

TAYLOR, A.L.; SASSER, J.N. Biology, identification and control of root-knot nematodes (Meloidogyne species). Raleigh: International Meloidogyne Project, NCSU \& USAID Coop. Publ., 1978. 111p. 